\title{
Pseudoanquilose da articulação temporomandibular devido ferimento de arma de fogo: abordagem cirúrgica de dois casos
}

\author{
Pseudo-ankylosis of the temporomandibular joint due to firearm injury: \\ a surgical approach of two cases \\ Falsa anquilosis de la articulación temporomandibular debido a la lesión de \\ arma de fuego: un enfoque quirúrgico de dos casos \\ Patrícia Rota BERMEJO \\ Juliana Coléte ZORZI \\ Gustavo Antonio Correa MOMESSO \\ William Phillip Pereira da SILVA \\ Leonardo Perez FAVERANI \\ Élio Hitoshi SHINOHARA
}

Departamento de Cirurgia e Clínica Integrada, Faculdade de Odontologia de Araçatuba,

Univ. Estadual Paulista (UNESP), Araçatuba, São Paulo, Brasil

\begin{abstract}
Resumo
A pseudoquililose da articulação temporomandibular (ATM) é uma condição rara muito menos frequente que as anquiloses intracapsulares (verdadeiras). A infecção, o trauma e a artrite são algumas das causas das limitações progressivas para a abertura da boca. O tratamento mais comum consiste em coronoidectomia unilateral ou bilateral. Este artigo tem como objetivo relatar dois casos clínicos de pacientes com pseudoanquilose da ATM devido a uma lesão causada pelo ferimento de arma de fogo, submetidos a coronoidectomia unilateral e remoção de projéteis de arma de fogo sob anestesia geral e acesso intraoral.
\end{abstract}

Descritores: Articulação Temporomandibular: Anquilose; Cirurgia Bucal.

\begin{abstract}
The pseudo-ankylosis of temporomandibular joint (TMJ) is a rare condition much less frequent than intracapsular (true) ankylosis. Infection, trauma and arthritis are some of the causes of progressive limitations for opening the mouth. The most common treatment consists of unilateral or bilateral coronoidectomy. This article aims to report two clinical cases of patients with ATM pseudo-ankylosis due to an injury caused by gunshot injury, subjected to unilateral coronoidectomy and removal of firearm projectiles under general anesthesia and intraoral access.

Descriptors: Temporomandibular Joint; Ankylosis; Surgery, Oral.

\section{Resumen}

La falsa anquilosis de la articulación temporomandibular (ATM) es una condición rara mucho menos frecuente que la anquilosis intracapsular (verdadera). La infección, el trauma y la artritis son algunas de las causas de las limitaciones progresivas para la apertura de la boca. El tratamiento más común consiste en coronoidectomía unilateral o bilateral. Este artículo tiene como objetivo relatar dos casos clínicos de pacientes con falsa anquilosis de la ATM debido a una lesión causada por la lesión de arma de fuego, sometidos a la coronoidectomía unilateral y remoción de proyectiles de arma de fuego bajo anestesia general y acceso intrabucal.

Descriptores: Articulación Temporomandibular; Anquilosis; Cirugía Bucal.
\end{abstract}

\section{INTRODUÇÃO}

A pseudoanquilose da articulação
temporomandibular (ATM) é uma condição rara muito menos frequente do que as anquiloses intracapsulares (verdadeiras) ${ }^{1}$. A infecção, o trauma e a artrite são algumas das causas das limitações progressivas para a abertura da boca, no entanto, o fator etiológico mais comum constitui patologias de junção extrínseca ${ }^{2}$. Esta condição é caracterizada por hipomobilidade mandibular, que pode ser variável do dano no movimento da lateralização ao trismo severo $^{3}$. O tratamento mais comum consiste em coronoidectomia unilateral ou bilateral. A intervenção deve ser realizada o mais cedo possível e a abordagem pode variar entre submandibular, periauricular ou intraoral ${ }^{3-5}$.

\section{CASO CLÍNICO}

\section{- Caso 1}

Paciente do sexo masculino, 23 anos procurou o Serviço de Cirurgia e Traumatologia Bucomaxilofacial, com queixa inicial de dificuldade de abertura bucal, o mesmo relatava ter sido vítima de ferimento por arma de fogo em face, há quatro anos atrás. Ao exame clinico, apresentava 17 $\mathrm{mm}$ de abertura bucal, com presença de uma cicatriz na região de pele em ângulo mandibular direito compatível com o orifício de entrada do projétil.
Radiograficamente apresentava fragmentos do projétil alojado em região de espaço pterigoideo lado esquerdo. O paciente foi submetido ao tratamento cirúrgico sob anestesia geral, com a realização de coronoidectomia unilateral e remoção dos fragmentos do projétil (Figuras $1 \mathrm{e}$ 2), com acessos intrabucais e foi submetido ao início do tratamento de fisioterapia no primeiro dia pós-operatório. Após o acompanhamento pós-operatório de 6 meses, o paciente apresentava uma abertura de boca de $40 \mathrm{~mm}$.

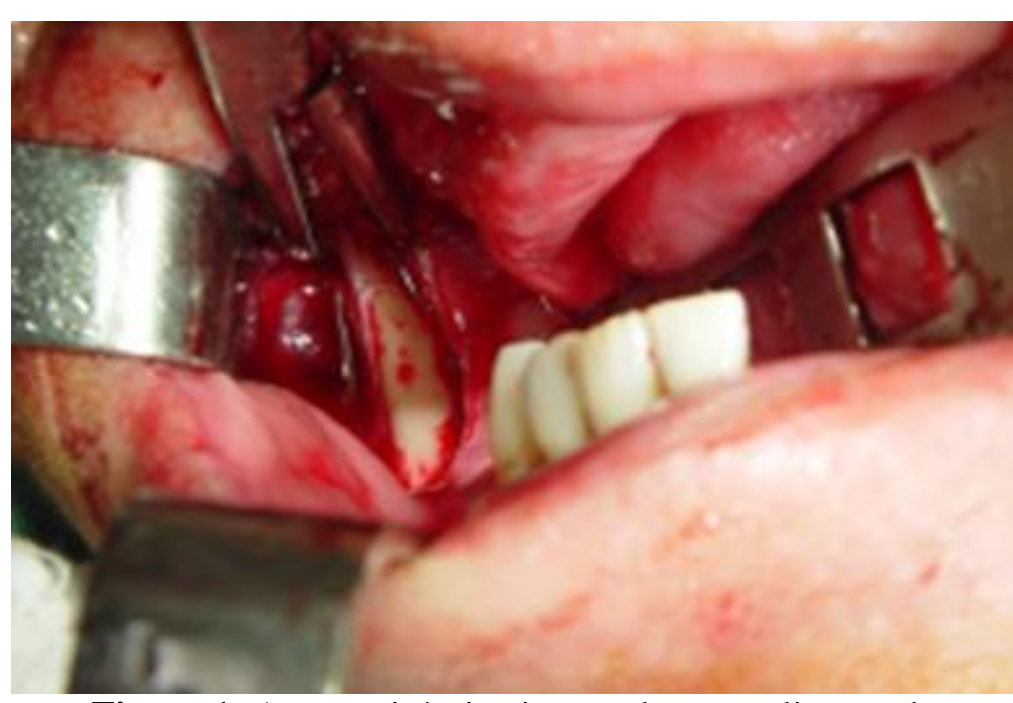

Figura 1: Acesso cirúrgico intraoral para realização da coronoidectomia. 


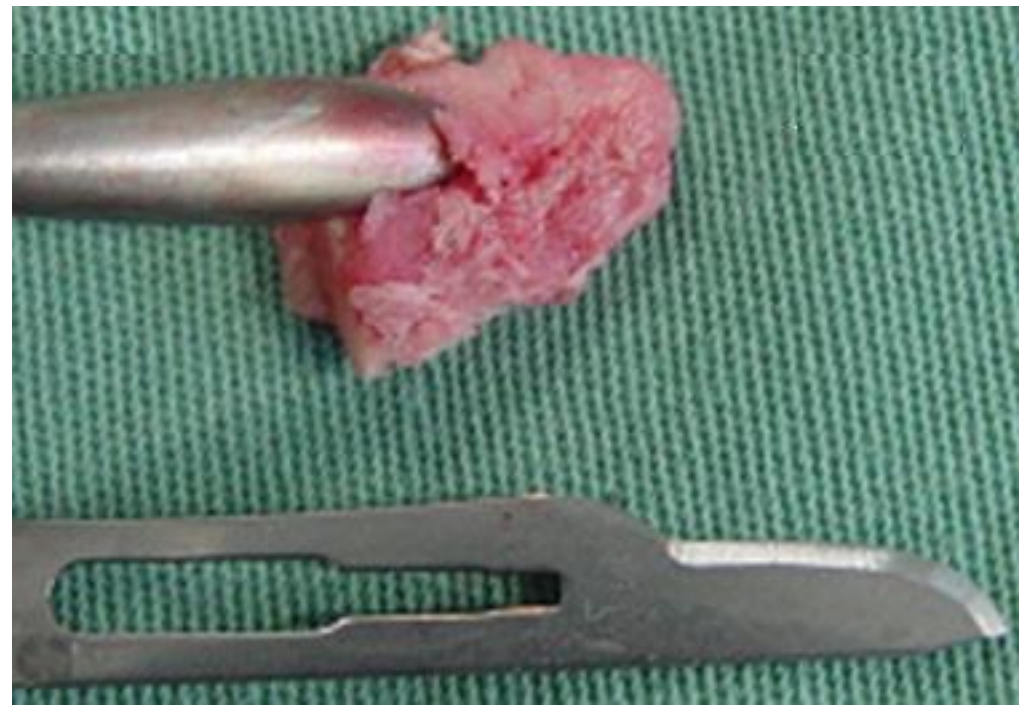

Figura 2: Fragmento ósseo do coronóide removido.

- Caso 2

Paciente do sexo feminino, 54 anos de idade apresentava queixa de dificuldade de abertura bucal e com histórico de ferimento por arma de fogo em face há dois anos. Apresentava ao exame clinico inicial, uma limitação de abertura bucal de $10 \mathrm{~mm}$, dois orifícios de entrada de projéteis de arma de fogo na região zigomática direita, e radiograficamente apresentava o alojamento do projetil em região zigomática direita. A paciente foi submetida ao tratamento cirúrgico sob anestesia geral, com realização da coronoidectomia unilateral (Figuras 3 e 4), remoção dos fragmentos do projétil e ao tratamento fisioterápico. Após 3 meses de acompanhamento pós-operatório, obteve uma abertura da bucal de $45 \mathrm{~mm}$.



Figura 3: Acesso cirúrgico intraoral para realização da coronoidectomia.



Figura 4: Fragmento ósseo do coronóide e projétil de arma de fogo removido.

\section{DISCUSSÃO}

A pseudoanquilose mandibular pode ser resultado de desordem muscular, óssea, neurológica ou psiquiátrica, ocasionadas devido infecções, traumas ou tumores ${ }^{6}$. A pseudoanquilose óssea ocorre quando há uma fusão dos processos ósseos articulares, levando o paciente sofrer perdas funcionais como mastigação, deglutição, fonação e o comprometimento estético ${ }^{3,7,8}$. Os presentes relatos de casos nos trazem a pseudoanquilose óssea devido a fusão dos processos coronoides ao arco zigomático decorrentes do trauma após o ferimento de arma de fogo. Os traumas por armas de fogo geralmente causam um grande dano as vítimas, seja decorrente a assaltos, acidentes ou tentativas de suicídios, o desafio no atendimento primário do paciente é o suporte avançado de vida no trauma, após a estabilização do paciente, as condutas empregadas variam de acordo com a intensidade do trauma ${ }^{9}$. Ao contrário da maioria dos relatos de casos na literatura, os quais trazem grandes destruições teciduais e fraturas, as lesões de baixas intensidades causadas por arma de fogo, podem não trazer riscos a vida do paciente, isso varia de acordo com o calibre da arma, o tipo do projétil e a distância do disparo com o alvo ${ }^{10,11}$. Devido sua posição ser protegida pelo arco zigomático e o complexo zigomático maxilar, as fraturas diretas do processo coronoide são raras, e traumas indiretos como descrito nos dois relatos de casos deste artigo, podem ocasionar sequelas como a pseudoanquilose, quando não tratado imediatamente ${ }^{12}$. O tratamento das fraturas do complexo zigomático ou do processo coronoide variam de acordo com o grau de deslocamento e sintomas clínicos, podendo variar em tratamento conservador, através do controle de dieta ao tratamento cirúrgico, seja redução e fixação da fratura ou a remoção do processo coronoide con,12,13. $^{7}$. A decisão de realizar intervenção cirúrgica na pseudo anquilose depende de fatores relacionados ao paciente, como problemas de abertura bucal, na mastigação, fonação e o impacto psicossocial ${ }^{14}$. Nos presentes casos, devido à demora de procura do tratamento de ambos os pacientes, dois e quatro anos após o trauma inicial, podemos notar que a evolução dos sintomas foi gradual e progressiva. O tratamento pode variar em coronoidectomia uni ou bilateral, com abordagem intra ou extraoral, como acessos pré auriculares ou submandibulares $2,3,8,13,15$, de acordo com a literatura, a eleição da coronoidectomia unilateral com uma abordagem intraoral foi realizada em ambos os pacientes, visando a menor morbidade cirúrgica e os melhores resultados estéticos. Além do tratamento cirúrgico, o controle pós-operatório e o tratamento de fisioterapia local imediato, como realizado já no primeiro dia pós-operatório, contribuem e garantem a restauração da abertura dos movimentos da boca e do maxilar, evitando a neoformação óssea, a formação de fibrose e cicatrização dos músculos ${ }^{7}$.

\section{CONSIDERACÕES FINAIS}

A avaliação inicial, o tipo de tratamento, conservador ou cirúrgico, bem como acompanhamento do paciente em consultas periódicas é fundamental para o prognóstico do paciente. Em casos de abandono ou recusa do tratamento, como os pacientes relatados, é recomendado o tratamento cirúrgico e fisioterápico imediato no pós-operatório para o tratamento tardio da pseudoanquilose.

\section{REFERÊNCIAS}

1. Rikalainen R, Lamberg MA, Tasanen A. Extra-articular fibrous ankylosis of the mandible after zygomatic fracture. J Maxillofac Surg. 1981; 9(2):132-6.

2. Hollins RR, Moyer DJ, Tu HK. Pseudoankylosis of the mandible after temporal bone attached craniotomy. Neurosurgery. 1988;22(1 Pt 1):137-9. 
3. Kumar P, Singh V, Agrawal A, Bhagol A, Bali R. Incrementsl increase in percentage mouth opening after coronoidectomy in temporomandibular joint ankylosis. Int J Oral Maxillofac Surg. 2015; 44(7):859-63.

4. Al-Saadi, Al Hashmi A. Outcomes of coronoidectomy as treatment of coronoid pseudoankylosis and hyperplasia: case series. Oral Maxilofac Surg. 2017; 46(Suppl 1):224.

5. Gaetti-Jardim EC, Oliveira JG, Mendonca JC. Bilateral temporomandibular joint ankylosis as sequel of bilateral fracture of the mandibular condyle and symphysis. Oral Maxilofac Surg. 2014; 18(1):39-42.

6. da Costa Araujo FA, Melo Barbalho JC, de Farias ON, Jr., de Vasconcellos RJ, do Egito Vasconcelos BC. Pseudo-ankylosis caused by osteoma of the coronoid process. Ann Maxillofac Surg. 2014; 4(2):208-10.

7. Baraldi CE, Martins GL, Puricelli E. Pseudoankylosis of the temporomandibular joint caused by zygomatic malformation. Int $\mathrm{J}$ Oral Maxillofac Surg. 2010;39(7):729-32.

8. Rikanailen R, Lamberg MA, Tasanen A. Extra-articular fibrous ankylosis of the mandible after zygomatic fracture. J Maxillofac Surg. 1981;9(2):132-6.

9. de Santana Santos T, Frota R, Martins-Filho PR, Vajgel A, de Albuquerque Maranhão-Filho AW, de Oliveira e Silva ED. Fracture of the coronoid process, sphenoid bone, zygoma, and zygomatic arch after a firearm injury. J Craniofac Surg. 2011;22(6):e34-7

10. Johnson J, Markiewicz MR, Bell RB, Potter BE, Dierks EJ. Gun orientation in self-inflicted craniomaxillofacial gunshot wounds: risk factors associated with fatality. Int J Oral Maxillofac Surg. 2012;41(8): 895-901.

11. Powers DB, Delo RI. Characteristics of ballistic and blast injuries. Atlas Oral Maxillofac Surg Clin North Am. 2013;21(1):15-24.

12. de Oliveira DM, Vasconcellos RJ, Laureano Filho JR, Cypriano RV. Fracture of the coronoid and pterygoid processes by firearms: case report. Braz Dent J. 2007;18:168-70.

13. Shen L, Li J, Li P, Long J, Tian W, Tang W. Mandibular coronoid fractures: Treatment options. Int J Oral Maxillofac Surg. 2013; 42(6):721-6.

14. Spijkervet FKL, de Bont LGM, Boering G. Management of pseudoankylosis of the temporomandibular joint: Report of cases. J Oral Maxillofac Surg. 1994; 52(11):1211-7.

15. Lehman H, Fleissig Y, Abid-el-raziq D, Nitzan DW. Limited mouth opening of unknown cause cured by diagnostic coronoidectomy: a new clinical entity? Br J Oral Maxillofac Surg. 2015; 53(3):230-4.

\section{CONFLITO DE INTERESSES}

Os autores declaram não haver conflitos de interesse.

\section{AUTOR PARA CORRESPONDÊNCIA}

\section{William Philip Pereira da Silva}

william_philip@hotmail.com

Submetido em 27/10/2017 Aceito em 07/12/2017 